\title{
Thickness Constraints for Topology Optimization Using the Fictitious Physical Model
}

$\operatorname{AUTHOR}(S):$

Yamada, Takayuki

\section{CITATION:}

Yamada, Takayuki. Thickness Constraints for Topology Optimization Using the Fictitious Physical Model. EngOpt 2018 Proceedings of the 6th International Conference on

Engineering Optimization 2019: 483-490

\section{ISSUE DATE:}

2019

URL:

http://hdl.handle.net/2433/236042

\section{RIGHT:}

This is a post-peer-review, pre-copyedit version of an article published in 'EngOpt 2018 Proceedings of the 6 th International Conference on Engineering Optimization'. The final authenticated version is available online at:

https://doi.org/10.1007/978-3-319-97773-7_43; The full-text file will be made open to the public on 14 September 2019 in accordance with publisher's 'Terms and Conditions for Self-Archiving'; この論文は出版社版でありません。引用の際 には出版社版をご確認ご利用ください。; This is not the published version. Please cite only the published version. 


\title{
Thickness Constraints for Topology Optimization Using the Fictitious Physical Model
}

\author{
Takayuki Yamada ${ }^{1}$ \\ Kyoto University, C3, Kyoto-Daigaku-Katsura, Nishikyo-Ku, Kyoto, 615-8504, Japan \\ takayuki@me.kyoto-u.ac.jp \\ orcid: 0000-0002-5349-6690
}

\begin{abstract}
Thickness constraint is an important geometrical constraint in topology optimization methods. I present a novel approach of the thickness constraint based on the Fictitious Physical Model (FPM). The FPM is formulated using the similarity of the dispersive coefficient in high order homogenization. The thickness constraint is represented using the solutions of the linear partial deferential equation system. Its design sensitivity is derived using the adjoint variable method. Numerical example is shown to confirm the validity and utility of the proposed method using the level set-based topology optimization method. The main advantage of the proposed method is the allowance of thickness constraint violations during the optimization procedure. Furthermore, the thickness is computed without computing minimum distances from the boundaries of target shape.
\end{abstract}

Keywords: geometrical shape feature, thickness, topology optimization, fictitious physical model

\section{Introduction}

Topology optimization has been widely used in industrial products, such as automobiles, trains and airplanes, because it is a powerful design tool to obtain creative design solutions with high performance. However, a design solution obtained by topology optimization requires an interpretation process by design engineers considering the manufacturing process. One of the most important manufacturability considerations in the design optimization process is considering the minimum and maximum thickness constraint. The minimum length scale constraint in topology optimization is proposed $[5,3]$ to avoid obtaining too complex structural members which may cause severe increase of manufacturing cost. The methods for maximum length scale constraint are also proposed [2]. However, these methods force design solution to fall into local optimal solution. That is, the initial configuration influences the obtained optimal solutions.

On the other hand, the concept of the fictitious physical model (FPM) [6] for representing geometrical constraint can be avoid such issues. Therefore, the main 
aim of this paper is to formulate a FPM for representing the thickness constraint. Additionally, the proposed FPM is applied to the topology optimization method. First, I formulate the FPM to represent thickness by the solutions of a linear partial differential equation. Additionally, its validity is discussed using a numerical example. Second, I briefly discuss a level set-based topology optimization method. Next, an optimization problem considering the thickness constraint is formulated by using the proposed FPM. Finally, a numerical example is provided to confirm the proposed topology optimization method.

\section{Formulation of the Fictitious Physical Model}

The basic concept of the fictitious physical model (FPM) is an evaluation method of target geometrical features by using the solution of a partial differential equation system. For instance, an area having very high stress may be predicted as a small shape feature, because the stress concentration is occurring around a small hole. However, the displacement field is not appropriate to evaluate the size of the material domain, because stress is dependent on much other information, such as boundary conditions and other domain shapes. In general, it is pretty difficult to precisely evaluate thickness or size of a structural member by using usual physical phenomena, because the physical features are dependent on much other information. Therefore, this paper presents a FPM and a thickness function $h_{f}$ representing a solution of the FPM.

First of all, we clarify the requirements of the thickness function $h_{f}$. The evaluation of thickness of a structural member must be satisfied as follows:

1. The thickness function $h_{f}$ is a monotonic function or linear function of the local thickness.

2. A parallel translated shape is equivalently evaluated to original shape. That is, the distribution of the function $h_{f}$ in a parallel translated shape is equivalent to distribution of the function $h_{f}$ in the original shape.

3. A rotated shape is equivalently evaluated to original shape. That is, the distribution of the function $h_{f}$ in a rotated shape is equivalent to rotated distribution of the function $h_{f}$ in the original shape.

Here, the local thickness is defined as the thickness of the target structural member at the local position. We note that the local thickness is not equivalent to local size. For example, a small hole of material domain is not small thickness. Of course, the small hole can be also evaluated by considering an opposite situation.

Next, we formulate the FPM for the thickness evaluation. We consider a reference domain $\Omega_{R}$ consisting of a material domain $\Omega$ and a void domain $\Omega_{R} \backslash \Omega$. We assume that the reference domain $\Omega_{R}$ is sufficiently huge to contain the target material domain. Here, we focus on the similarity of the geometrical features of dispersive coefficient in periodic homogenization. As proofed in Allaire and Yamada's work [1], dispersive coefficient values in $k$ times scaled unit cell are equivalent to $k^{2}$ times values of the original dispersive coefficient. Based 
on this feature and the similarity, the fictitious physical model is formulated as follows:

$$
\left\{\begin{array}{lr}
-\operatorname{div}\left(\tilde{a} \nabla \chi_{i}-e_{i} \mathbf{1}_{\Omega}\right)+\alpha\left(1-\mathbf{1}_{\Omega}\right) \chi_{i}=0 & \text { in } \quad \Omega_{R} \\
\chi_{i}=0 & \text { on } \quad \partial \Omega_{R}
\end{array}\right.
$$

where $\chi_{i} \in H^{1}\left(\Omega_{R}\right)$ are $i$-th fictitious state variables, $e_{i}$ are the canonical bases of $\mathbb{R}^{d}, \tilde{a}>0$ is the diffusion coefficient and $\alpha$ is the damping coefficient. The characteristic function $\mathbf{1}_{\Omega} \in L^{\infty}\left(\Omega_{R}\right)$ is defined as

$$
\mathbf{1}_{\Omega}(\boldsymbol{x})= \begin{cases}1 & \text { for } \quad \boldsymbol{x} \in \Omega \\ 0 & \text { for } \quad \boldsymbol{x} \in D \backslash \Omega .\end{cases}
$$

We introduce a parameter $a$ for the diffusion coefficient satisfied as $\tilde{a}:=a h_{0}^{2}$, where the characteristic length in the FPM is defined as the target thickness $h_{0}>0$. The damping coefficient must be set to a large value to force the values of state variables $\chi_{i}$ into zero almost everywhere in the void domain. We remark that the details of the formulation are found based on the author's intuition and trial and error approach.

Next, we define the thickness function $h_{f}\left(\{\chi\}_{1 \leq i \leq d}\right)$ as follows:

$$
h_{f}\left(\{\chi\}_{1 \leq i \leq d}\right):=\left\{\frac{1}{h_{0}}\left(\sum_{i=1}^{d} \frac{\partial \chi_{i}(\boldsymbol{x})}{\partial x_{i}}\right)^{-1}-h_{0} a\right\} \mathbf{1}_{\Omega},
$$

where we set the damping coefficient $\alpha$ as follows:

$$
\alpha:=\frac{4}{a}
$$

Then, the parameter of the proposed FPM is the non-dimensional diffusion parameter $a$ only. The parameter $a$ should be set sufficiently small, because the damping coefficient $\alpha$ is set to keep off effect from the surrounding domain and boundary of the reference domain $\partial \Omega$. The detailed features of the thickness function $h_{f}\left(\{\chi\}_{1 \leq i \leq d}\right)$ are discussed in the next subsection.

Additionally, to avoid numerical singularity, I introduce the function $f_{h}$ defined as follows:

$$
f_{h}\left(\{\chi\}_{1 \leq i \leq d}\right):=h_{0}^{2}\left(\sum_{i=1}^{d} \frac{\partial \chi_{i}(\boldsymbol{x})}{\partial x_{i}}\right)
$$

That is, thickness should be evaluated by the function $f_{h}$ on behalf of the thickness function $h_{f}$ in numerical computations. I note that the function $f_{h}$ is equivalent to

$$
\frac{1}{\frac{h_{h}}{h_{0}}+a} .
$$

in the material domain $\Omega$. Therefore, the function $f_{h}$ has monolithic relationship in $\Omega$.

I note that the sensitivity for the topology optimization is easily derived because the thickness is formulated by the solutions of the standard linear partial differential equation. 


\section{Numerical Validation of the Proposed FPM}

The two-dimensional reference domain $\Omega_{R}$ shown in the Figure 1 is considered. The gray area and white area represent material domain and void domain, re-

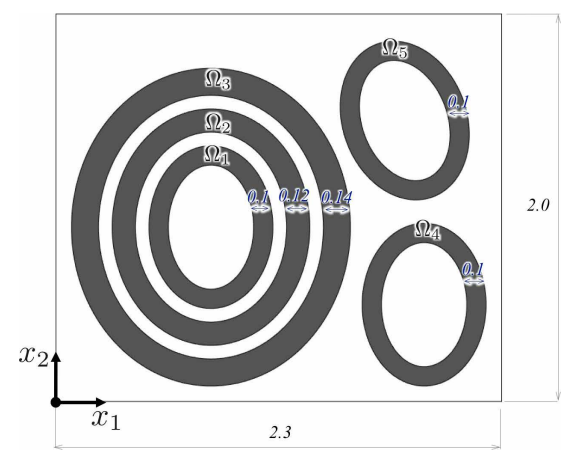

Fig. 1. Material distribution for the validation

spectively. The parameters of the fictitious physical model are set to $h_{0}=0.1$ and $a=0.2$. The domain is discretized using triangular elements and we use $\mathbb{P} 2$ finite elements whose maximal length is 0.03 . Then, the obtained distributions of the fictitious variables and the thickness function are shown in Figure 2.

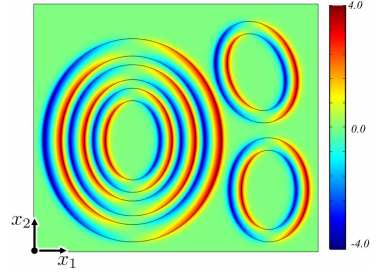

(a) The state variable $\chi_{1}$

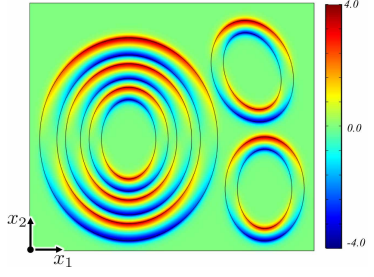

(b) The state variable $\chi_{2}$

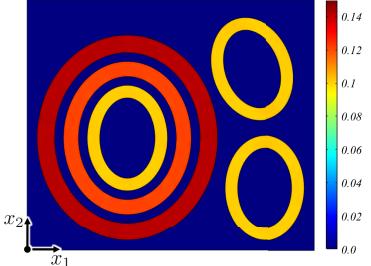

(c) The thickness function $h_{f}\left(\chi_{1}, \chi_{2}\right)$

Fig. 2. Distribution of the fictitious variables and the thickness function

Here, let us check the validity of the proposed model based on the obtained results qualitatively. To begin with, we focus on the domains $\Omega_{1}, \Omega_{2}$ and $\Omega_{3}$, which are ring shape with different thickness. We remark that a ring shape has a constant thickness. Therefore, the thickness function $h_{f}\left(\chi_{1}, \chi_{2}\right)$ must be valued as a constant value in each ring shape. Based on Figure 2, we confirm that the value of the thickness function $h_{f}\left(\chi_{1}, \chi_{2}\right)$ in the ring shapes is constant. 
Additionally, these values monotonously decrease with increasing thicknesses. Therefore, the proposed model satisfies the condition 1 defined in the above subsection.

Next, we focus on the domains $\Omega_{1}$ and $\Omega_{4}$, which are the same ring shape and located in different positions. That is, the domain $\Omega_{4}$ is a parallel translated shape from domain $\Omega_{1}$. The values of those thickness functions $h_{f}\left(\chi_{1}, \chi_{2}\right)$ are equivalent, as shown in Figure 2. Therefore, the proposed model satisfies the condition 2 defined in the above subsection.

In the third step, we focus on the domains $\Omega_{1}$ and $\Omega_{5}$, which are the same ring shape. These locations and angles are different. That is, the domain $\Omega_{5}$ is rotated and parallel translated from domain $\Omega_{1}$. The values of those thickness functions $h_{f}\left(\chi_{1}, \chi_{2}\right)$ are also equivalent, as shown in Figure 2. Therefore, the proposed model satisfies the condition 3 defined in the above subsection.

\section{Formulation of Topology Optimization Problem with Thickness Constraint}

The basic concept of the topology optimization is replacement of a structural design optimization problem with a material distribution problem, which is represented by fixed design domain and the characteristic function $\mathbf{1}_{\Omega}$. Then, a standard topology optimization problem is formulated as follows:

$$
\begin{array}{ll}
\inf _{\mathbf{1}_{\Omega}} & F\left(\mathbf{1}_{\Omega}\right)=\int_{D} f(\boldsymbol{x}) \mathbf{1}_{\Omega} \mathrm{d} \Omega \\
\text { subject to: } & G\left(\mathbf{1}_{\Omega}\right)=\frac{\int_{\Omega} \mathbf{1}_{\Omega} \mathrm{d} \Omega}{\int_{\Omega} \mathrm{d} \Omega}-V_{\max } \leq 0
\end{array}
$$

where $F, G$ and $V_{\max }$ are objective function, constraint function concerning the volume constraint and upper limit of the volume constraint, respectively. The thickness constraint should be imposed for every point in the material domain as follows:

$$
h_{\min } \leq h(\boldsymbol{x}) \leq h_{\max } \quad \text { in } \quad \Omega
$$

where $h_{\min }$ and $h_{\max }$ are the lower and upper limit of the thickness constraint, respectively. The constraint is replaced by the following equation because the thickness is evaluated by the function $f_{h}$ in the FPM using the lower limit $f_{h \text { min }}$ and upper limit $f_{h_{\max }}$.

$$
f_{h_{\min }} \leq f_{h}\left(\{\chi\}_{1 \leq i \leq d}\right) \leq f_{h_{\max }} \quad \text { in } \quad \Omega
$$

Additionally, the thickness constraint is evaluated by the integral in the material domain $\Omega$ to replace a scalar functional by the distributed function in the optimization process. 
In the level set-based topology optimization method $[7,4]$, the structural boundaries are represented by the iso-surface of the level set function $\phi(\boldsymbol{x})$ :

$$
\begin{cases}1 \leq \phi(\boldsymbol{x})>0 & \text { for } \quad \boldsymbol{x} \in \Omega \backslash \partial \Omega \\ \phi(\boldsymbol{x})=0 & \text { for } \quad \boldsymbol{x} \in \partial \Omega \\ 0>\phi(\boldsymbol{x}) \leq-1 & \text { for } \quad \boldsymbol{x} \in D \backslash \Omega\end{cases}
$$

The characteristic function is redefined by using the level set function $\phi(\boldsymbol{x})$ as follows:

$$
\mathbf{1}_{\phi}:= \begin{cases}1 & \text { for } \quad \phi(\boldsymbol{x}) \geq 0 \\ 0 & \text { for } \quad \phi(\boldsymbol{x})<0\end{cases}
$$

Then, the topology optimization problem with the volume constraint and thickness constraint is formulated as

$$
\begin{aligned}
& \inf _{\phi} \\
& F(\phi)=\int_{D} f(\boldsymbol{x}) \mathbf{1}_{\phi} \mathrm{d} \Omega \\
& \text { subject to: } \\
& G(\phi)=\frac{\int_{\Omega} \mathbf{1}_{\phi} \mathrm{d} \Omega}{\int_{\Omega} \mathrm{d} \Omega}-V_{\max } \leq 0 \\
& f_{h_{\text {min }}} \leq f_{h}\left(\{\chi\}_{1 \leq i \leq d}\right) \leq f_{h_{\max }} \\
& -\operatorname{div}\left(\tilde{a} \nabla \chi_{i}-e_{i} \mathbf{1}_{\phi}\right)+\alpha\left(1-\mathbf{1}_{\phi}\right) \chi_{i}=0 \\
& \text { in } \Omega_{R} \\
& \chi_{i}=0 \\
& \text { on } \partial \Omega_{R}
\end{aligned}
$$

I note that the reference domain $\Omega_{R}$ is defined to contain the fixed design domain $D$.

In the level set-based topology optimization, the shape and topology changes during the optimization procedure are represented as an evolution of the level set function $\phi$. That is, introducing fictitious time $t$, the shape and topology evolution is obtained by solving the following reaction-diffusion equation:

$$
\frac{\partial \phi}{\partial t}=-K^{2}\left(L^{\prime}-\tau \nabla^{2} \phi\right)
$$

where $K$ is a coefficient of proportionality and $L^{\prime}$ is design sensitivity including constraints, i.e., the topological gradient of the Lagrangian of the optimization problem. The regularization parameter $\tau$ is set to a small value. The details are discussed in Yamada's work [7].

\section{Numerical Example of the Proposed Topology Optimization Method}

A numerical example is provided to confirm the utility of the proposed method. In the example, the isotropic linear elastic material has Young's modulus = 210GPa and Poisson's ratio $=0.31$. The regularization parameter $\tau$ is set to 


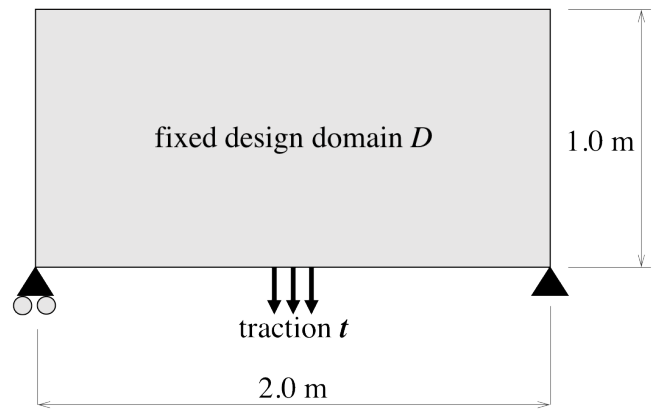

Fig. 3. Fixed design domain and the boundary conditions

$1 \times 10^{-5}$. The upper limit of the volume constraint $G_{\max }$ is set to 0.5 . Figure 3 shows the fixed design domain and boundary conditions. The traction is set to $\boldsymbol{t}=(0,1000) N$. Here, we consider the minimum mean compliance problem. That is, the objective function is set to

$$
F:=\int_{\Gamma_{t}} \boldsymbol{t} \cdot \boldsymbol{u} \mathrm{d} \Gamma
$$

where $\Gamma_{t}$ and $\boldsymbol{u}$ are boundary imposed traction $\boldsymbol{t}$ and the displacement field, respectively. The fixed design domain is discretized using a structural mesh and a four-node quadrilateral plane stress element whose length is $0.01 \mathrm{~m}$. Figure 4 shows obtained optimal configurations. Cases (a) and (b) are an obtained configuration without constraint and considering upper limit of the thickness, respectively. As shown in Figure 4, both results are smooth and similar feature.

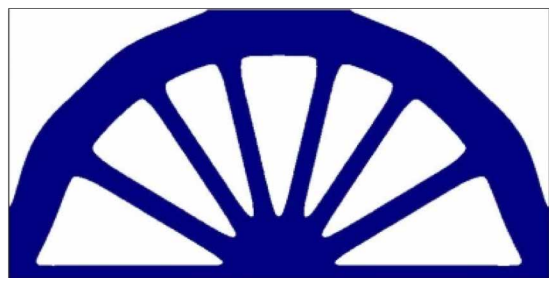

(a) Thickness constraint free

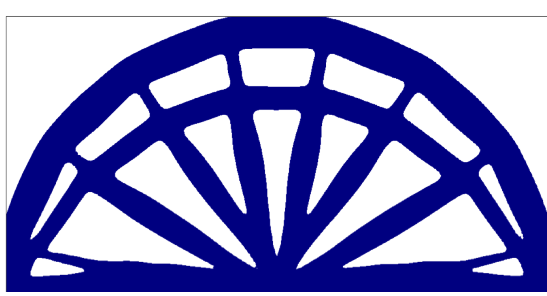

(b) Considering thickness constraint

Fig. 4. Obtained optimal configurations

Although Case (a) includes thick parts, Case (b) does not include any thick part. Therefore, the proposed method provides appropriate optimal configurations considering the thickness constraint. 


\section{Conclusions}

This paper proposed a new method of thickness constraint for topology optimization using the FPM. First, the FPM is formulated to represent the thickness of the material domain. The topology optimization problem with the thickness constraint is formulated using the proposed FPM. I confirmed the usefulness of the proposed method by the numerical example.

\section{References}

1. Allaire G, Yamada T (2018) Optimization of dispersive coefficients in the homogenization of the wave equation in periodic structures. Numerische Mathematik, in press, https://hal.archives-ouvertes.fr/hal-01341082

2. Guest JK (2009) Imposing maximum length scale in topology optimization. Structural and Multidisciplinary Optimization 37(5):463-473, doi:10.1007/ s00158-008-0250-7

3. Guest JK, Prévost JH, Belytschko T (2004) Achieving minimum length scale in topology optimization using nodal design variables and projection functions. International Journal for Numerical Methods in Engineering 61(2):238-254, doi: 10.1002/nme.1064

4. Otomori M, Yamada T, Izui K, Nishiwaki S (2015) Matlab code for a level set-based topology optimization method using a reaction diffusion equation. Structural and Multidisciplinary Optimization 51(5):1159-1172, doi:10.1007/s00158-014-1190-z

5. Poulsen TA (2003) A new scheme for imposing a minimum length scale in topology optimization. International Journal for Numerical Methods in Engineering 57(6):741-760, doi:10.1002/nme.694

6. Sato Y, Yamada T, Izui K, Nishiwaki S (2017) Manufacturability evaluation for molded parts using fictitious physical models, and its application in topology optimization. The International Journal of Advanced Manufacturing Technology 92(14):1391-1409, doi:10.1007/s00170-017-0218-0

7. Yamada T, Izui K, Nishiwaki S, Takezawa A (2010) A topology optimization method based on the level set method incorporating a fictitious interface energy. Computer Methods in Applied Mechanics and Engineering 199(45):2876-2891, doi:10.1016/j. cma.2010.05.013 